\title{
Rough set based QoS enabled multipath source routing in MANET
}

\author{
Prathviraj N. ${ }^{1}$, Santosh L. Deshpande ${ }^{2}$ \\ ${ }^{1}$ Manipal School of Information Sciences, Manipal Academy of Higher Education, Manipal INDIA \\ ${ }^{2}$ Department of PG Studies Visvevaraya Technological University, Balagavi, INDIA
}

\begin{tabular}{l}
\hline Article Info \\
\hline Article history: \\
Received Jul 6, 2019 \\
Revised Oct 12, 2019 \\
Accepted Oct 21, 2019 \\
\hline
\end{tabular}

\section{Keywords:}

Cross layer

Manet

Multipath

Quality of service

Rough set theory

\begin{abstract}
The single constrained Quality of Service (QoS) routing in Mobile Ad-hoc NETwork (MANET) is disastrous in consideration of MANET characteristics, inference, collision and link failure as it maintains a single path. The QoS enabled routing yields better packet delivery and maintains consistency among nodes in the network by incorporating multi-constrained and multipath routing. The Dynamic Source Routing (DSR) is best suited source routing algorithm to maintain multipath information at the source node, but performance degrades with larger number of mobile nodes. Multilayer mechanism should be incorporated to maintain QoS metric information spreads across multiple layers of TCP/IP protocol stack. The proposed multipath QoS enabled source routing provides balanced routing by making use of all these features. The imprecise decision making strategy called Rough Set Theory (RST) is used at destination node for decision making. The Route REQuest (RREQ) messages coming from different routes are filtered by considering the QoS metrics of each and every route by making use of RST. The Route REPly (RREP) messages are generated and delivered to the source node for filtered RREQ messages. The proposed routing algorithm will reduce load on the network by reducing number of control messages exchanged for route establishment. This will evenly distribute load among all the nodes and it also avoid the scenarios like few nodes starved for resources. Finally, multipath routing always provides alternate routing option in case of route failure.
\end{abstract}

Copyright $\odot 2020$ Institute of Advanced Engineering and Science. All rights reserved.

\section{Corresponding Author:}

Prathviraj N.,

Manipal School of Information Sciences,

Manipal Academy of Higher Education,

Manipal, India.

Email: praj151986@gmail.com

\section{INTRODUCTION}

The wireless medium and randomness feature of nodes makes Mobile Ad-hoc NET (MANET) work in a different way compared to other wired and wireless networks. MANET is spread across indefinite boundary and is open to interference from unwanted signals. MANET works without any centralized communication system, here each and every mobile node communicates directly with Independent Basic Service Set (IBSS) [1-2]. The IBSS works in a distributed manner by synchronizing all nodes to a common clock without any infrastructure support. The routing uses deterministic approach for delivering the packets effectively and routing algorithms used in these types of dynamic network should adapt quickly to nondeterministic nature of the MANET. Many traditional ad-hoc routing protocols have been proposed till now, such as DSR, Ad Hoc On-Demand Distance Vector (AODV), Temporally Ordered Routing Algorithm (TORA) etc. MANETs practical implementation and use in the real world multimedia application demands more than what traditional best effort protocols are able to deliver [3]. The multimedia application always 
demands certain guaranteed delivery of information with respect to certain QoS metrics such as bandwidth, delay, jitter, packet loss rate, hop count and path reliability [4]. Enabling QoS in routing should consider multi-layer design methods [5], here input from physical, data link and network layer are integrated in order to satisfy the needs of resource demanding applications.

The path reliability here in MANET depends on the link stability of each link from source to destination because of varying mobility of the mobile nodes. Very frequent movement of mobile nodes with different speed results in link breaks and network partition [6]. In both the cases the source node needs to rediscover a path from source to destination. The rediscovery process increases the routing delay if the network is very dense, which results in slow convergence thus reduces the throughput. The established QoS routing depends on one or two fixed static QoS metrics; most of the time relay on hop count, delay and bandwidth. Along with this QoS routing comes with additional overhead of disseminating QoS information while routing [7]. This makes the selected best path runs out of QoS resources and leads to amplification of issues with respect to MANET. The QoS provisioning is very important aspect in any novel routing algorithm; in order to achieve this multi path, multi-layer and stability features should be incorporated. The multipath aims to balance utilization of resources among the nodes. The available multiple paths between source and destination will be add-on in consideration of dynamic nature of MANET [8]. The QoS enabled routing requires collection of present scenario from the lower MAC and physical layers. The input collected from lower layers will give the real time QoS requirement and it can be used for designing the QoS enabled routing protocol. Routing the packets in consideration of stability of the path will give protection from non-availability of the path. The stability of the path can be downsized to stability of link involved in the path construction [9]. Here stability is dependent on parameters like signal strength, relative speed between nodes, energy etc.

Multi path routing is about maintaining multiple paths between source and destination; it will consider QoS aspects as well as dynamic and non-deterministic nature of MANET. So ultimately multi-path routing overcomes problems with respect to single path routing but it comes with the overhead of computing and maintaining more than one path. On-demand nature of source routing protocol overrides the requirement of periodic exchange of route advertisement packet for neighbor detection present in the other protocol $[10,11]$. The DSR is a representative of on-demand routing protocols, where multiple paths can be returned to the source nodes. The generally used standard single constrained QoS routing algorithm makes an extra overhead compared to other best-effort routing algorithm, since extra information being communicated to maintain QoS. These factors lead to a higher drain of resources on mobile nodes. Numerous attempts have been made to optimize multi-constraint QoS routing with consideration of two or more fixed QoS parameters. The limited applicability in terms of fixed QoS parameters in such methods leads to performance degradation in other QoS aspects. The genetic algorithm enabled multi constrained QoS routing [12] proposed to reserve resources required by collecting all nodes state information. Collecting and maintaining the state information is an overhead in this methodology. Practically there is still space for a novel multi-constrained QoS routing which incorporates multiple QoS parameters with minimum overhead. A novel routing should balance and extend the lifetime of nodes in the network by balancing the load among all nodes.

The Rough Set Theory (RST) is an excellent mathematical tool to build the decision model from the large set of inconsistent data with multiple attributes [13, 14]. Extracting decision model in terms of rules from inconsistent data is a crucial task without considering any additional information on data. This concept can be considered as another approach to deal with vagueness and imprecise, unlike dealing only with precise data set in fuzzy set theory. Suppose, QoS parameter of the different route is considered as a set of objects U called universe and an equivalence relation $R \in U \times U$. The proposed multipath QoS enabled source routing overcomes the drawback identified in single constrained and multi constrained QoS routing. The decision rules derived from RST are applied to limit the control message exchanged in route discovery process. The decision rules are used to filter the RREQ message and RREP messages will be generated only for selected RREQ messages. Since decision rules are framed considering all QoS aspects, selected routes will be able to balance QoS resource in network. The rest of the research article spans across different sections. Section 2 gives reflection on literature study. The proposed multipath QoS enabled source routing is discussed under section 3. In section 4 experimental setup and results are discussed. Finally section 5 concludes the research article.

\section{REFLECTION ON LITERATURE}

The delay, energy, throughput, packet loss rate and jitter are the commonly used metrics by MANET applications to specify QoS requirements to any routing protocol. In accordance with this, these are frequently used as constraints on route discovery and selection [15]. Each and every QoS metric is identified 
by expected reference point, which will be considered as a constraint in QoS routing. For example Minimum Required Throughput or Capacity (b/s), Maximum Tolerable Delay (s), Jitter, Maximum Tolerable Packet Loss Ratio (PLR). The application running on MANET typically requires more than one of the above mentioned QoS requirements.

The routing protocols adopted in MANET provide different routing strategy. Few routing protocols considers QoS aspects in route discovery stage, few other routing protocols do not consider QoS [16]. Along with this some routing protocols are working with MAC layer and others are not. Based on these prerequisites QoS protocols can be classified into three categories: first, reserving quantified resource and availability of those resources in any network conditions, called as pseudo-hard QoS. TDMA is one of the examples for such contention free MAC solution. Providing guaranteed QoS service in MANET scenario is extremely hard. Second, reserving the resources and likely utilize lesser resources than reserved, called as soft-QoS. These routing strategies are dependent on contended MAC protocol, so work on available resources. Third, without reserving the resources attempting to forward route request message under favorable scenarios, called as no QoS. These routing strategies are independent of MAC protocol and do not promising any QoS guarantee. The aim of such protocols is to provide immediate delivery without promising anything [17, 18]. The QoS routing protocols can be classified under different categories for the better understanding of how these protocols are working to enable QoS in routing [19]. So the QoS protocols can be classified under 3 different categories: first, based on the QoS approach incorporated in routing. QoS approach again can be classified under two different categories. Coupled QoS approach, in which QoS provisioning is closely associated with routing and any change in routing may fail to provision guaranteed QoS. The decoupled QoS approach does not depend on any particular routing protocol. Second, QoS provisioning can also be classified according to layer on which these protocols are provisioning QoS aspects. The protocols working on MAC layer make use of random back off and channel sensing mechanism, these protocols will be suitable for real time traffic expecting guaranteed bandwidth. Third, QoS provisioning is not dependent on any routing algorithm or layers. This is considered as QoS framework for providing better service in terms of packet delivery.

The deployment of QoS aspects in MANET routing focus only throughput and delay to some extent. Even though throughput and delay are prominent QoS aspects to be considered, relying only on these leads to compromise in other QoS parameter. The characteristics of MANET shift the focus from single constrained QoS to multi-constrained QoS. The complexity involved in multi constrained makes QoS routing NP-complete problem [20]. The QoS guarantee mechanism incorporates input from all layers of TCP/IP, here every layer is responsible for corresponding QoS metric [21, 22]. The network layer is having the impact on very critical QoS metric like multimedia QoS and network resources. The data link layer is responsible for setting up the priorities for the business flow, to ensure compliance of end to end QoS and scheduling the channel selection. The physical layer is responsible for controlling the throughput, error rate and sending rate to achieve QoS requirement. The different business requirement demands different categories of QoS requirement and these QoS requirements can be satisfied from different layers of the protocol stack [23, 24]. So, each layer of the protocol stack should be considered independently to meet the business requirement of various QoS demanding application. The load balancing among all nodes in the MANET improves performance of the node in all QoS aspects. Load balancing mitigates network congestion and increases the overall network throughput. It also processes every packet and every flow for every destination independently. The existing multi path routing protocols utilizes all available path to deliver packets to the destination. Here load balancing is implemented [25] by sharing the load among all available paths.

\section{QOS ENABLED MULTIPATH SOURCE ROUTING}

QoS enabled multipath routing is intended for deriving multiple reliable routes from source to destination with consideration of multiple QoS aspects. The proposed routing algorithm should consider multiple QoS metric such as minimum bandwidth, balanced energy consumption, minimum end to end delay and maximum packet delivery fraction. The availability of QoS metric related information is limited to different layers of protocol stack. So, the proposed algorithm should perform the multi-layer mechanism to collect QoS metric information from different layers. The mobile node participating MANET routing maintains route cache, it keeps track of available active route information from that particular source node to different destination. The mobile node initiating packet delivery has to first check in its route cache for availability of active route information. If active route is not available then route discovery process is initiated by the source node. The route discovery process works in two stages: route request and route reply. 
The packet generated for route request message consists of different QoS metric information collected from different layer in addition to regular route request (RREQ) message. The destination node receives RREQ message from different route with varying QoS metric information. The rough set theory decision rules are applied to filter the RREQ message and for the filtered RREQ message RREP message will be generated. The RREP message delivered to the source node consists of route information to the destination.

\subsection{QoS enabled multipath}

The multiple paths between source and destination will be discovered in the route discovery process of DSR. These available multiple paths will be used if there is congestion on the path with first priority or if the path is unavailable due to movement of nodes. So, multipath routing enhances the performance of packet delivery by compensating the unpredictable nature of MANET. Benefits of multipath routing comes with some drawbacks like computation overhead and extra routing table space to maintain multiple paths. The flexible nature of on demand source routing makes it simpler to compute and maintain multiple paths. This will lessen the overhead of multipath.

\subsection{QoS enabled cross layer}

The QoS guaranteed routing in ad-hoc network takes input from all the layers of TCP/IP protocol stack, each and every layer contributing to collect input for QoS parameters. So, QoS enabled multipath algorithm is a multi-layer design which incorporates input from all layer of the TCP/IP protocol stack. The proposed algorithm collects QoS requirements from application layer, end-to-end throughput and latency from transport layer, throughput from data link layer, bandwidth and energy from physical layer.

\subsection{QoS Enabled route reliability}

The path reliability is defined as the ability of the path to successfully deliver data packets to the destination. Predicting the ability of path in dynamic scenario like MANET is difficult job. The ability of the path is dependent on resources available on all intermediate nodes; accumulation of this information will be the deciding factor for the reliability of the path. Here resources are nothing but QoS parameters, which includes different classes of parameters like additive, comparative and multiplicative. In multi parameters decision making significance of all the parameters has to be taken into consideration separately.

The significance of parameter is evaluating how much that parameter is important to successfully transmit data. The significance of the particular QoS parameter is calculated by evaluating impact of negating that QoS from routing information table. Let QoS and route be a set of condition and decision attributes. Let energy be a condition attributes, i.e., energy $\in$ QoS. The representation of degree of dependency of condition with dependency is shown as $(Q o S$, route $)$. Difference in eliminating energy from condition leads to lower the degree of dependency. This can be represented as follows:

$$
\sigma(\text { energy })=\gamma(\text { QoS }, \text { route })-\gamma(\text { QoS }-\{\text { energy }\} \text {, route })
$$

The difference will yield the significance of attribute energy and it is represented using $\sigma($ energy). QoS enabled route parameters

\subsection{Bandwidth estimation}

Make sure that channel used for packet forwarding is free and channel utilization is estimated by the bandwidth. The availability of bandwidth information is from data link layer, multi-layer mechanism is incorporated to fetch the bandwidth information. The available bandwidth at the time of arrival of RREQ message is calculated by considering the consumed bandwidth from the raw bandwidth. The comparative strategy is used to compute the bandwidth in the RREQ message traversal, whichever link is having low bandwidth availability is stored in the RREQ.

\subsection{Delay estimation}

The delay involved in delivering the packet from source to destination is accumulation of a delay in all intermediate nodes participating in the traversal of packets. Estimation of the delay is based on the round trip time and queuing delay. The queuing delay is impacted by number of packets already arrived and waiting in a buffer, i.e. load on the node. The round trip time is accumulation of processing delay and transmission delay. The processing delay is time required to extract packet header information to get to know where to direct the packet. The transmission delay is time required to transmit and to receive the packet. The additive strategy of all such delays in the packet traversal is maintained in the packet header. 


\subsection{Energy estimation}

The significance of energy parameter in QoS routing very high, so energy estimation should be done very carefully. The remaining energy should be considered out of initial energy after consuming the energy for other packet traversal. The energy parameter of the arrival RREQ message is compared with remaining energy of the node, whichever is low updated in the RREQ message. The nodes remaining energy is updated by considering energy consumed for transmission and reception of the current packet traversal.

\subsection{Route finding process}

The node which intend to send the message, check for available route information in the route cache. If routes are available sender will make use of the existing routes for message delivery. If no routes are available sender makes an attempt to initiate route discovery process. In the route discovery process two types of messages exchanged between source and destination: RREQ and RREP. Nodes in the MANET prone to changes its position dynamically, restricts all routes to expiration time. The correction of routes from the missing link will initiate route maintenance phase, here missing link will be identified and reported back to the source node.

\subsubsection{Route request}

The RREQ packet consists of IPV4 address of source and destination node, unique route identification number, route record to keep track of all intermediate nodes it passes through and for QoS parameters. The intermediate node checks the incoming RREQ packets route identification and make sure that same packet is not received through other nodes. If it has already received simply discard the packet, otherwise compare the QoS parameters make the updation in RREQ.

\subsubsection{Rough set theory}

The routing problems in dynamic nature MANET identify the requirement of dynamically changing QoS parameters in routing condition balance the trade-off between QoS parameter. The RSES is used to analyse the values of QoS parameters with different techniques like data discretization, reduction, decision rules and prediction. The significance of the particular attribute or condition values will be calculated based on the impact of that attribute in the decision rules. The Rough Set Exploration System (RSES) is used for data exploration, discretization, reduction and decision making. It is an open source tool helps in understanding tabular data set generated from stack parameter configuration and makes decision with respect to reason for variation in different QoS parameters. In order to ensure good decision has been made accuracy and coverage of the decision will be displayed for all QoS parameters.

The discretization is the partition of set of values into subset based on the cuts fulfilling some standard condition. Here partition is done to make sure that if subset falls under certain decision rule than all QoS values that comes under particular subset will be in aligned with the respective decision rule. The Figure 1 shows the discretization of values into multiple subsets based on the cut set from the original decision table. The cut set will give the boundary for the partition. So the discretization will be helpful to take the decision rule with respect to subset and for all the values of subset decision rule will be applied.

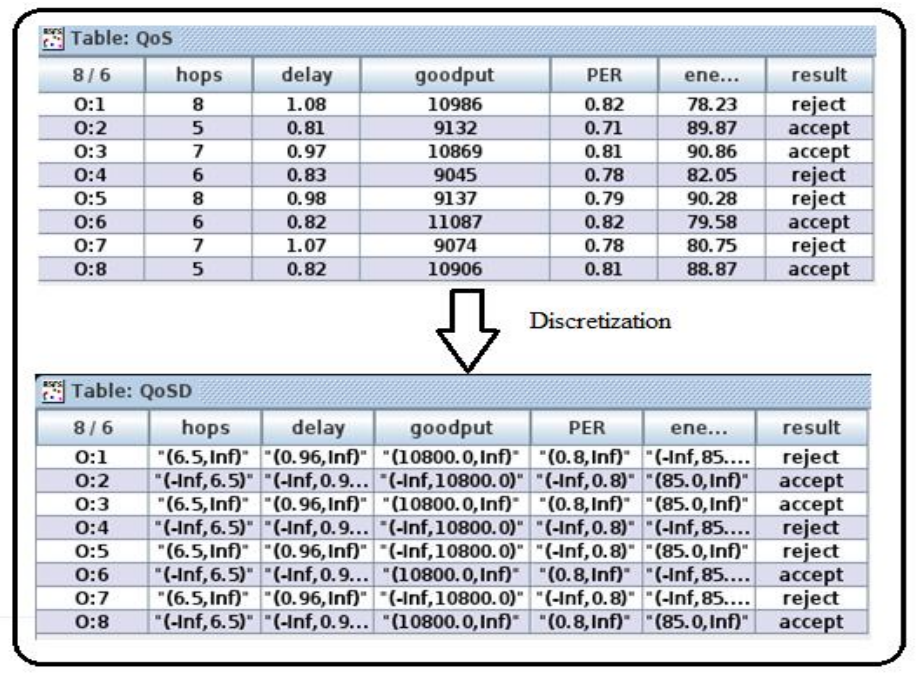

Figure 1. Transformation of original data set into discretized information 
The decision rule table consists of set of rules which specifies the different QoS condition to be considered before forwarding packet. The RSES will come up with set of decision rules as shown in Figure 2, based on the discretized decision table and significance of the attribute. The coverage of the decision rule should be all rows of the decision table. The impact of energy parameter on the decision rule is 0.5 , based on energy parameter decision can be taken on the decision attribute. The removal of energy leads to loss of 50\% consistency of decision rule. The impact of all other parameter like hops, goodput, delay, PER is 0.25 means removal of other parameter leads to loss of $25 \%$ consistency of decision rule.

\begin{tabular}{|c|c|c|}
\hline \multicolumn{3}{|c|}{ Rule set: QOSD } \\
\hline$(1-16)$ & Match & Decision rules \\
\hline 1 & 2 & $\left(\right.$ hops $\left.="(6.5, \ln f)^{\prime \prime}\right) \&($ energy $="(-\ln f, 85,0) ") \Rightarrow>($ resultt $=\{$ reject[2] $\})$ \\
\hline 2 & 2 & $\left(\right.$ delay $\left.==^{\prime}(0.96, \ln f)^{\prime}\right) \&\left(\right.$ energy $\left.==^{\prime}(-\ln f, 85.0)^{\prime}\right) \Rightarrow($ result $=\{$ reject $[2]\})$ \\
\hline 3 & 2 & (hops="(-Inf,6.5)")\&(energy =" (85.0,Inf)") $\Rightarrow($ result $=\{$ accept $[2]\})$ \\
\hline 4 & 2 & 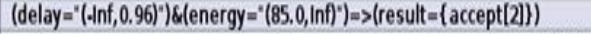 \\
\hline 5 & 2 & (goodput $="(10800.0$, Inf)" $) \&\left(\right.$ energy $\left.="(85.0, \operatorname{lnf})^{\prime}\right) \Rightarrow>($ result $=\{$ accept $[2]\})$ \\
\hline 6 & 2 & (PER=" $\left.(0,8, \ln f)^{\prime \prime}\right) \&($ energy $="(85,0$, inf)" $) \Rightarrow$ (result=\{accept $\left.[2]\}\right)$ \\
\hline 7 & 2 & (goodput="(-.nff,10800.0)")\&(energy $\left.="(-\ln f, 85.0)^{\prime \prime}\right) \Rightarrow>($ result $=\{$ reject[2]\}) \\
\hline 8 & 2 & (PER="(-Inf,0.8)")\&(energy="(-Inf,85.0)") $\Rightarrow($ result=\{reject[2]\}) \\
\hline 9 & 2 & (hops $\left.="(6,5, \operatorname{lnf})^{\prime \prime}\right) \&(g 00 d p u t="(-\ln f, 10800,0) ") \Rightarrow($ result $=\{$ reject[2]\}) \\
\hline 10 & 2 & (hops $="(6.5, \ln f)^{\prime)} \&\left(P E R="(-\ln f, 0.8)^{\prime}\right) \Rightarrow>($ result $=\{$ reject[2]\}) \\
\hline 11 & 2 & 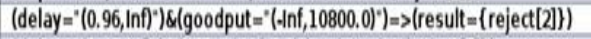 \\
\hline 12 & 2 & 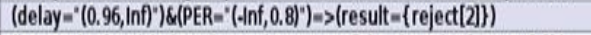 \\
\hline 13 & 2 & (hops="(-Int,6.5)")\&(goodput="(10800.0, Inf)" $) \Rightarrow($ result $=\{$ acceptt $[2]\})$ \\
\hline 14 & 2 & (hops $\left.="(-\operatorname{Inf}, 6.5)^{\prime \prime}\right) \&\left(\right.$ PER $\left.="(0.8, \operatorname{Inf})^{\prime}\right) \Rightarrow>($ result $=\{$ accept $[2]\})$ \\
\hline 15 & 2 & (delay ="(-Inf,0,96)")\&(goodput=" $(10800.0$, Inf)" $) \Rightarrow($ result = $\{$ accept[2]\}) \\
\hline 16 & 2 & 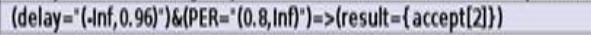 \\
\hline
\end{tabular}

Figure 2. Rule set generated from RST

\subsubsection{Route reply}

The RREP packet is generated for RST filtered paths by the destination node, which will place all relevant information in reply message. The RREP will take same path which will earlier used by RREQ, since this route information is stored in the packet. Otherwise traversal of the RREP packet will takes place by referring to cache of the intermediate node and this route information in the route record. Once source node receives such RREP packet through different path, now source node knows how to communicate with the destination.

\subsubsection{Route Maintenance}

Since source node receives multiple RREP messages and multiple paths are maintained, route maintenance in the proposed algorithm is simpler. To get to know existing path is not available for message transfer two types of feedback message is exchanged, i.e. Route Error (RERR) and Acknowledgment (ACK). The existence of the path is confirmed to neighboring nodes by communicating ACK packet on delivery of the packet to next hop. Whenever ACK packet is not received RERR packet is initiated and delivered to the source node. Alternate path maintained by source node through multipath routing comes to help and new packet is send through alternate path.

\section{EXPERIMENTAL SET UP AND RESULT}

The simulation environment developed with variable number of mobile nodes like $25,30 \ldots 75$ within a $1000 \mathrm{~m} \times 1000 \mathrm{~m}$ area. Each mobile node has its own radio propagation range and these nodes are placed with in their range. The $5 \mathrm{sec}$ is set as pause time and mobility speed is established between $2 \mathrm{~m} / \mathrm{s}$ to $12 \mathrm{~m} / \mathrm{s}$ for these mobile nodes. To analyze the performance of MQSR over the DSR different application level traffic and transport layer protocols were incorporated. The experimentation setup includes CBR and FTP as application traffic, here CBR uses UDP and FTP uses TCP as transport layer protocol.

The experimental results are analyzed with respect to packet drop, delay and throughput for variable number of mobile nodes. The Figure 3 depicts the packet drop variation for MQSR and DSR routing for variable number of mobile nodes. The packet drop of DSR gradually increases with respect to number of mobile nodes but MQSR controls the packet drop even with more number of nodes. The Figure 4 depicts variation of delay in packet arrival with respect to MQSR and DSR for different size of network. 
The performance of DSR gradually degrading in terms of delivering packet on time as there is an increase in number of nodes participating in the network. On the other hand MQSR steadily delivering the packet without much delay even with more number of nodes. The Figure 5 represents the throughput variation with respect to MQSR and DSR for variable number of mobile nodes. Like packet drop and delay throughput also degrading as there is increase in number of nodes for DSR routing algorithm. On the other hand MQSR shows not much drop in throughput with higher number of mobile nodes.

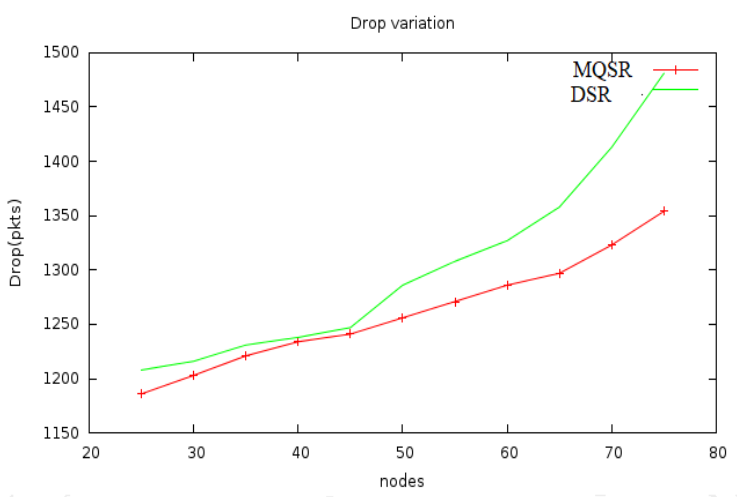

Figure 3. Packet drop variation

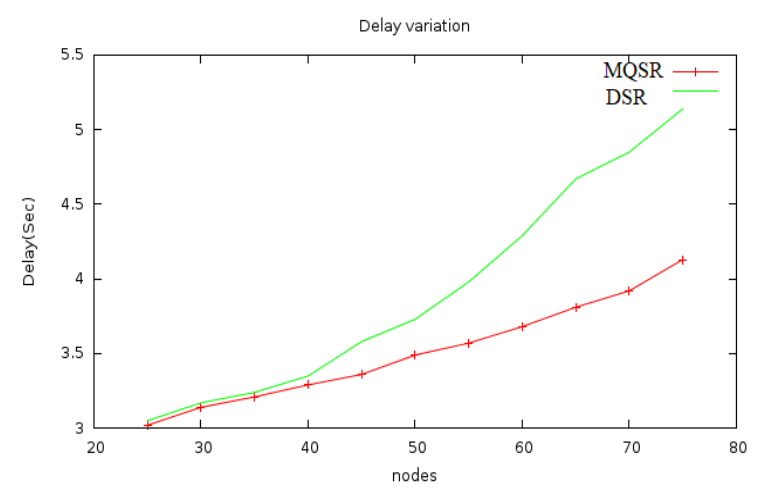

Figure 4. Delay variation

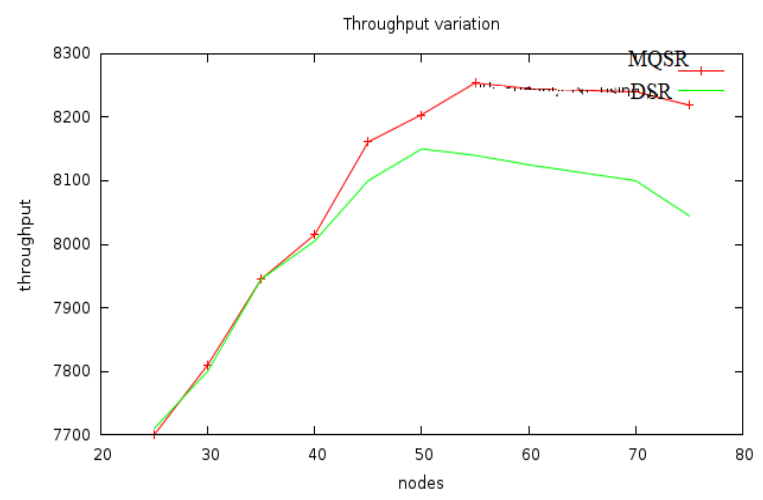

Figure 5. Throughput variation

\section{CONCLUSION}

The proposed multipath QoS enabled source routing algorithm incorporates multipath and multilayer mechanism into DSR. The extended version of DSR enhances the performance of packet delivery by taking into consideration of all QoS metric from different layers of protocol stack. The RST based route filtering at the destination node and generation of the RREP message only for filtered route makes the balanced usage of nodes over the network. It also overrides the drawback of DSR specifically with more number of nodes. The performance of MQSR compared against DSR with respect to packet drop, delay and throughput. The experimental results clearly shows better performance in MQSR against DSR especially in larger networks.

\section{REFERENCES}

[1] Ashtiani H, Moradi H, Pour P, Nikpour, "A Survey of MANET Routing Protocols in Large-Scale and Ordinary Networks," Global Journal of Computer Science and Technology, Vol. 10(13):39-46, 2010.

[2] Cho AM, Moe A A., "Energy efficient multipath routing for mobile ad hoc networks," International Journal of Information Technology Modeling and Computing, Vol. 2(3):11-18, 2014.

[3] D. B. Johnson and D. A. Maltz, "Dynamic source routing in ad hoc wireless networks, in mobile computing," edited by Tomaz Imielinski and Hank Korth, Chapter 5, Kluwer academic publishers, pp. 153-181, 1996. 
[4] Georgios Parissidis, "Multi-path Routing Protocols in Wireless Mobile Ad Hoc Networks": A Quantitative Comparison Springer-Verlag Berlin Heidelberg, 2009.

[5] A. M. Satyabrata Ch., "QoS Issues in Ad Hoc Wireless Networks," IEEE Commun. Mag., pp. 142-148, 2001.

[6] Gizem, Aksahya \& Ayese, Ozcan , "Multipath Routing with Load Balancing in Wireless Ad Hoc Networks", Coomunications \& Networks, Network Books. ABC Publishers. ROMA I N G R O L EAU, 2009.

[7] Primo Skraba, Hamid Aghajan and Ahmad Bahai, "Cross-Layer Optimization for High Density Sensor Networks," USA: Springer Berlin / Heidelberg. Vol. 17, 2004.

[8] Pawlak Z., "Rough Sets," International Journal of Computer and Information Sciences, vol. 11, no. 5, pp. 341-356, 1982.

[9] Pradeep, B.S. and Soumya, S., "A new method for load balancing and QOS in on demand protocols - in the MANET's perspective," International Journal of Advanced Networking and Applications, Vol. 1, No. 4, pp.275-281, 2010.

[10] Prathviraj N and Santosh L Deshpande, "Rough Set Approach for Solving Mulit Constrained QoS Routing Problems in MANET," IEEE WISPNET , pp. 1193-1196, 2016.

[11] Prathviraj N, Santosh L Deshpande, "Rough Set-Based Analysis of Multilayer Stack Parameter on QoS of MANET," Lecture Notes on Data Engineering and Communications Technologies , vol. 28, pp. 217-229, 2019.

[12] S.-J. Lee, C.-K. Toh, and M. Gerla, "Performance Evaluation of TableDriven and On-Demand Ad Hoc Routing Protocols," in Proceedings of IEEE PIMRC’99, Osaka, Japan, pp. 297-301, 1999.

[13] S. R. Das, R. Castaneda and J. Yan, "Simulation Based Performance Evaluation of Mobile, Ad Hoc Network Routing Protocols," ACM/Baltzer Mobile Networks and Applications (MONET) Journal, pages 179-189, 2000.

[14] Suresh HN, Varaprasad G, Jayanthi G., "Designing energy routing protocol with power consumption optimization in MANET," IEEE Transactions on Emerging Topics in Computing. Vol. 2(2):192 -197, 2014.

[15] S.Venkatasubramanian and Dr.N.P.Gopalan, "A Quality of Service Architecture for Resource Provisioning and Rate Control in Moblie Ad-hoc Network," International Journal of Ad hoc, Sensor \& Ubiquitous Computing (IJASUC) Vol.1, No.3., 2010.

[16] Varshavsky, A. Reid, B. de Lara, "A cross-layer approach to service discovery and selection in MANETs," Canada: Mobile Adhoc and Sensor Systems Conference (IEEE), 2005.

[17] K. Jayabarathan, J., Avaninathan, S., Savarimuthu,R. "QoS enhancement in MANETs using priority aware mechanism in DSR protocol," J Wireless Com Network, 2016.

[18] R. Asokan, A. Natarajan, "Performance Evaluation of Energy and Delay Aware Quality of Service(QoS) Routing Protocols in Mobile Adhoc Networks," International Journal of Business Data Communications and Networking, 4 , pp. 52-63,2008.

[19] H Shen, L Zhao, "ALERT: an anonymous location based efficient routing protocol on MANETs," IEEE Trans. Mob. Comput. 12(6), 1079-1093, 2013.

[20] XM Zhang, Y Zhang, F Yan, A Vasilakos, "Interference-based topology control algorithm for delay-constrained mobile ad hoc networks," IEEE Trans. Mob. Comput. 14(4), 742-754, 2015.

[21] SS Chaudhari, RC Biradar, "Survey of bandwidth estimation techniques in communication networks," Wirel. Pers. Commun. 83(2), 1425-1476, 2015.

[22] S. Fu and Y. Zhang, "CRAWDAD data set due/packet-delivery (v. 201503-30)," http://crawdad.org/due/packetdelivery/.

[23] S. Fu and Y. Zhang, "Experimental Study for Multi-layer Parameter Configuration of WSN Links," IEEE 35th International Conference on Distributed Computing Systems, pp. 369-378, 2015.

[24] V.V Mandhare and R.C. Thool, "Improving Qos of Mobile Ad hoc Network using Cache update scheme in Dynamic Source Routing protocol," 7th international conference on communication, computing and virtualization, Vol(79), pp.692-699. 2016.

[25] Yuh-Shyan Chen, Shin-Jr Jan and Ming-Chin Chuang, "A Shoelace-Based QoS Routing Protocol for Mobile Ad Hoc Networks Using Directional Antenna,” Wireless Personal Communications, SpringerLink., 2009.

\section{BIOGRAPHIES OF AUTHORS}

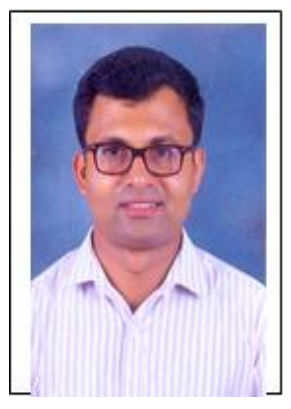

Prathviraj N. received his B.E in Computer Science and Engineering and MTech in Software Engineering from Visvesvaraya Technological University Belagavi, India, in 2009 and 2012 respectively. He is currently pursuing his PhD from Visvesvaraya Technological University under the guidance of Dr Santosh Deshpande. His research interest includes ad-hoc networks, wireless sensor networks, rough set theory. He is working as an Asst.Professor in Manipal School of Information Science, Manipal Academy of Higher Education Manipal, India. 


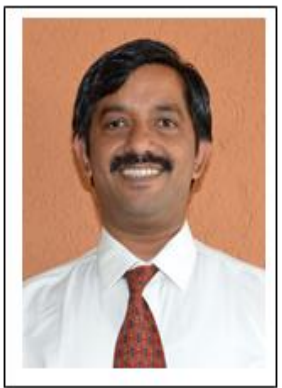

Dr Santosh L. Deshpande, received his B.E in Electronics and Communication Engineering from Shivaji University Kolhapur in 1996 and M.Tech in Computer Engineering from National Institute of Technology Karnataka in 2005. He obtained his Ph.D from J NTU Hyderabad in 2011. His research interest includes ad-hoc network, network security and wireless sensor network. Presently he is working as Professor and Head in Dept. of PG Studies VTU Belagavi, India. 